\title{
Early decompressing craniectomy in patients with traumatic brain injury and cerebral edema
}

\author{
Jiang-Biao Gong, Liang Wen, Ren-Ya Zhan, Heng-Jun Zhou, Fang Wang, Gu Li, Xiao-Feng Yang \\ Department of Neurosurgery, First Affiliated Hospital, College of Medicine, Zhejiang University, \\ Hangzhou 310003, China
}

\begin{abstract}
Background: Decompressing craniectomy (DC) is an important method for the management of severe traumatic brain injury (TBI).

Objective: To analyze the effect of prophylactic DC within 24 hours after head trauma TBI.

Methods: Seventy-two patients undergoing prophylactic DC for severe TBI were included in this retrospective study. Both of the early and late outcomes were studied and the prognostic factors were analyzed.

Results: In this series, cumulative death in the first 30 days after DC was 26\%, and 28 (53\%) of 53 survivors in the first month had a good outcomes. The factors including Glasgow Coma Score (GCS) score at admission, whether the patient had an abnormal pupil response and whether the midline shift was greater than 5 mm were most important prognostic factors for the prediction of death in the first 30 days and the final outcome at 6 months after DC.

Conclusion: Prophylactic DC plays an important role in the management of highly elevated ICP, especially when other methods of reduction of ICP are unavailable.
\end{abstract}

Keywords: Decompressing craniectomy, outcome, traumatic brain injury

Decompressing craniectomy (DC) is an important method for the management of severe traumatic brain injury (TBI), especially when patients develop refractory intracranial hypertension for which conservative methods are ineffective. Although there are still debates about its effect on improving patients' outcome, DC is widely performed for the management of TBI in medical institutes throughout the world.

At present, the European Brain Injury Consortium and Brain Trauma Foundation guidelines for severe traumatic brain injuries referred to DC as a secondtier therapy for refractory intracranial hypertension that does not respond to conventional therapeutic measures [1, 2]. Commonly, in most studies, surgical decompression is performed under the guidance of intracranial cerebral pressure (ICP) monitoring, and be used to reduce ICP after lack of effectiveness of other methods. By contrast, prophylactic or primary DC is performed very early, even without measuring ICP, or before the application of other methods for reducing ICP. This does not follow current guidelines

Correspondence to: Professor Xiao-Feng Yang, Department of Neurosurgery, First Affiliated Hospital, College of Medicine, Zhejiang University, Hangzhou 310003, China.

E-mail:jhw_hz@163.com
[3]. This type of prophylactic DC is performed frequently for the management of severe TBI. It would already be planned before or during the evacuation of mass lesions to present brain swelling $[3,4]$.

Clinical studies investigating the outcome of patients after DC demonstrate mortality rates of between $18 \%$ and $90 \%$ [5-10]. However, the heterogeneous character of the patient population, including different surgical procedures, different age groups, and changes in therapeutic concepts, especially whether this surgical decompression is performed as a prophylactic or secondary form, make it impossible to compare mortality figures among these studies and evaluate this surgical procedure.

This study focused on outcomes after undergoing prophylactic early DC in patients with severe TBI during the first 24 hours after TBI. We analyzed prognostic factors in this population and the complications secondary to this prophylactic surgical decompression.

\section{Methods \\ Patients}

We reviewed the clinical courses of consecutively admitted patients presenting to the First Affiliated 
Hospital, College of Medicine, Zhejiang University. Patients included were admitted from 2003 to 2008. Inclusion criteria were as follows: (1) patients who were admitted to this institution for severe TBI; (2) computed tomography (CT) imaging showed intracranial hematoma with or without the appearance of diffuse brain swelling; and (3) patients admitted to this institution underwent DC within $24 \mathrm{~h}$ after TBI as a prophylactic procedure. The indication of prophylactic DC included: (1) the patient had a severe head injury (a GCS score $\leq 8$ when admitted to our hospital) and was comatose; (2) the patient had an acute intracranial hematoma that needed surgical evacuation, and was expected to have severe cerebral edema after the operation. Exclusion criteria were as follows: (1) patients who were younger than 16 years; (2) prior neurologic conditions (including TBI) with residual disability or requiring ongoing medical care; (3) primary brain stem injury seen on initial CT scans.

Included patients were divided into two subgroups according to the outcome assessed at 6 months after the DC (Poor outcome, GOS $\leq 3$; Good outcome, GOS $\geq 4$ ).

The research protocol was approved by the Clinical Research Ethics Committee of our university.

\section{Prophylactic DC}

In this series, DC was performed as a prophylactic surgical therapy during the first 24 hours after TBI. DC included hyperventilation, barbiturates, therapeutic hypothermia, and ICP monitoring. Ventricular cerebrospinal fluid (CSF) drainage was not used before this surgical decompression. However, dehydration through mannitol or furosemide was used in some cases. All patients had intracranial hematoma after TBI disclosed by CT scanning, and this study did not include patients who underwent DC for simple diffuse brain swelling. DC was performed as a standard trauma craniotomy (large fronto-temporoparietal decompressive, $12 \mathrm{~cm} \times 15 \mathrm{~cm}$ cranial defect) for lesions confined to one cerebral hemisphere, and a bilateral frontal craniectomy from the floor of the anterior cranial fossa to the coronal suture of the pterion or bilateral standard trauma craniotomy for lesions located diffusely. Moreover, DC was performed together with augmentative duraplasty using temporal fascia or artificial meninges in all patients.

\section{Statistical analysis}

Differences between the two groups were compared using independent Student $t$ tests for continuous variables, Pearson chi-square tests, or Fisher's exact test for ranked variables. Data are presented as mean \pm standard deviation (mean \pm S.D.) and analyzed using SPSS version 13.0 for Windows (SPSS, Chicago, IL). $P<0.05$ was considered as statistically significant.

\section{Results \\ Patient population}

Seventy-two patients were included in this study. Demographics are shown in Table 1.

\section{Outcome}

After the first month following DC, nineteen patients died, and forty patients remained either in a vegetative state (nine patients), or severely disabled state (thirty-one patients). The thirty-day mortality was $26 \%$. Survivors were followed for 6 months after DC to determine their GOS. There were 2 patients who died during the follow-up beyond the first month after DC, and the overall mortality rate was $29 \%$. Finally, 28 patients had good outcomes (GOS 4 and GOS 5), and the remaining 44 patients had poor outcomes, including 21 patients who died, 3 patients remained in a vegetative state (GOS 2), and 20 severely disabled patients (GOS 3).

\section{Complications}

Contralateral intracranial hematoma developed in 6 patients (8\%), and reoperation was performed in 5 patients (Figure 1). All of these contralateral hematomas developed within 48 hours after DC. During the acute phase another frequent complication was brain herniation through cranial defects (Figure 2) for which CT imaging demonstrated evidence of increased swelling and contusion of the cortex underlying the edge of the cranial defect. Nine patients (13\%) develop this complication (a mean of 46 hours after DC). Thirteen patients (18\%) developing subdural effusions (Figure 3), 15 patients (21\%) experienced posttraumatic shunt-dependent hydrocephalus, and 3 (4\%) suffered posttraumatic epilepsy requiring antiepileptic agents.

\section{Prognostic Factors}

The potential factors which may be related to the outcome, included the patients' age, gender, GCS score at admission, whether or not the patient had an abnormal pupil response, and the degree of midline shift. 
Table 1. Patients’ demographics and GCS score at admission

\begin{tabular}{|c|c|}
\hline Number & 72 \\
\hline \multicolumn{2}{|l|}{ Sex } \\
\hline Male & 47 \\
\hline Female & 25 \\
\hline \multicolumn{2}{|l|}{ Age } \\
\hline Average (years old) & $46 \pm 16$ \\
\hline$<20$ & 1 \\
\hline 20-39 & 25 \\
\hline $40-60$ & 29 \\
\hline$>60$ & 17 \\
\hline \multicolumn{2}{|l|}{ Mechanism of TBI } \\
\hline Traffic accident & 59 \\
\hline Falling & 11 \\
\hline Violence & 2 \\
\hline \multicolumn{2}{|l|}{ GCS score } \\
\hline Average & $5 \pm 1$ \\
\hline $3-5$ & 44 \\
\hline $6-8$ & 28 \\
\hline$>8$ & 0 \\
\hline \multicolumn{2}{|l|}{ Intracranial lesion } \\
\hline Cerebral contusions & 72 \\
\hline Extradural hematoma & 8 \\
\hline Subdural hematoma & 60 \\
\hline Intracerebral hematoma & 37 \\
\hline Combined hematoma ${ }^{\mathbf{\wedge}}$ & 20 \\
\hline \multicolumn{2}{|c|}{ Outcomes (assessed by GOS score) } \\
\hline 1 & 21 \\
\hline 2 & 3 \\
\hline 3 & 20 \\
\hline 4 & 18 \\
\hline 5 & 10 \\
\hline
\end{tabular}

$\mathbf{\Lambda}$ = the patient who had more than one intracranial hematoma

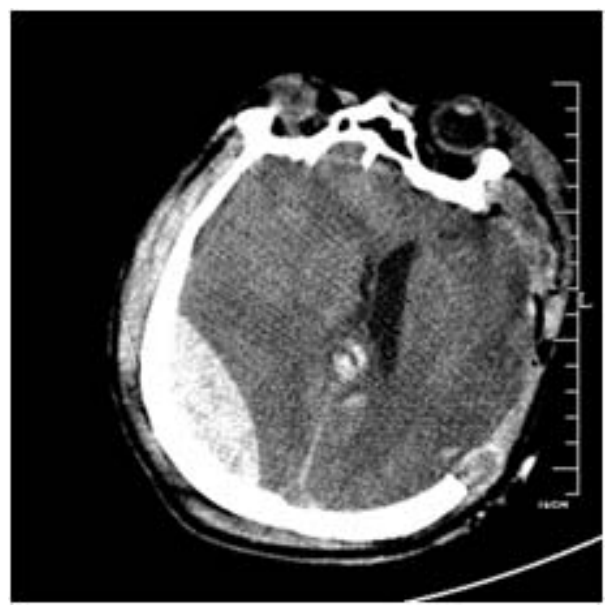

Figure 1. Contralateral acute extradural hematoma secondary to decompressive craniectomy 


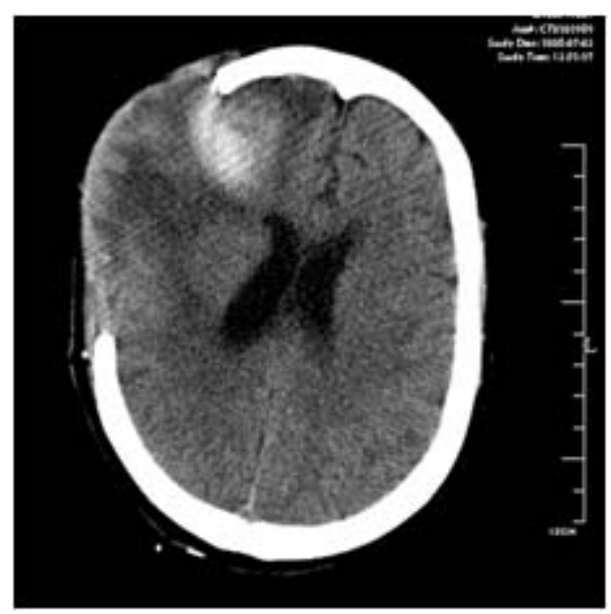

Figure 2. Brain herniation through cranial defect and hemorrhage at the edge of cranial defect

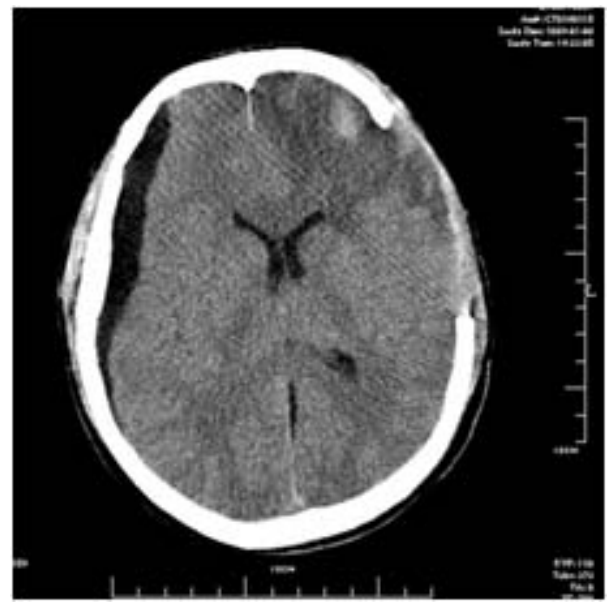

Figure 3. Contralateral subdural effusion secondary to decompressive craniectomy

Prognostic Factors for Early Outcome (30 days after DC)

Nineteen patients died at the time of 30 days after DC, and 53 patients survived. Between the two populations, the factors including GCS score at admission, whether the patient had abnormal pupil response, and whether the midline shift was greater than $5 \mathrm{~mm}$ were significantly different (Table 2).

It seems that a lower GCS score, abnormal pupil response, and midline shift that was greater than $5 \mathrm{~mm}$ were related to the outcome of death. However, in this series the patients' sex and age were not statistically different between the two populations.

Table 2. Predictors of death in the first 30 days after DC

\begin{tabular}{lllllll}
\hline & Number & GCS $^{\Delta}$ & Age $^{\Delta}$ & $\begin{array}{l}\text { Sex } \\
\text { (male/female) }\end{array}$ & $\begin{array}{l}\text { Abnormal` } \\
\text { pupil response }\end{array}$ & $\begin{array}{l}\text { Midline shift } \\
>\mathbf{5 ~ m m ~}\end{array}$ \\
\hline Group 1 & 19 & $4.0 \pm 0.8$ & $50.0 \pm 16.4$ & $15 / 4$ & $16 / 3(84 \%)$ & $18 / 1(96 \%)$ \\
Group 2 & 53 & $5.4 \pm 1.1$ & $44.9 \pm 15.3$ & $32 / 21$ & $19 / 34(36 \%)$ & $33 / 20(62 \%)$ \\
$P$ & & $<0.001$ & 0.239 & 0.171 & $<0.001$ & 0.008 \\
\hline
\end{tabular}

Group 1= population of patients who died in the first 30 days after DC, Group 1 = population of patients who survived in the first 30 days after DC, removal of mass lesion (no/yes), abnormal pupil response (yes/no), midline shift $>5$ mm (yes/no), $\Delta$ = independent Student $t$ test, $\boldsymbol{\Delta}$ = Pearson chi-square 


\section{Prognostic Factors of Late Outcome (6 months after DC)}

According to the outcome assessed 6 months after DC, the patients were divided to two subgroups: patients with good outcome $(\mathrm{n}=28)$ and those with poor outcome $(n=44)$. Similarly, it seems that the factors including GCS score at admission, whether the patient had an abnormal pupil response, and whether the midline shift was greater than $5 \mathrm{~mm}$ were related to poor outcomes (Table 3).

\section{Discussion}

Cerebral edema is a pathophysiological change following head trauma. It would contribute to elevation of ICP and reduction on cerebral perfusion pressure (CPP), which would successively cause detrimental effects on cerebral oxygen metabolism and can lead to catastrophic sequels. The association of elevated ICP caused by cerebral edema leading to poor outcomes in head trauma is well known [11-13]. Controlling the elevated ICP is the core principle for management of TBI. DC, which has been used for this aim for a long time, is a surgical procedure in which part of the skull is removed to allow room for swelling of the brain to expand without being constricted. In most studies, it would be performed following ICP monitoring as a second-tier method for controlling refractory intracranial edema and hypertension $[7,14,15]$. It may then be a desperate last method after exhaustion of others. Under these circumstances, DC is performed as a secondary separate surgical procedure in an attempt to reduce ICP and improve CPP by allowing the swollen brain to expand beyond the confines of dura and skull.

Primary or prophylactic DC is defined as a procedure with the initial evacuation of an intracranial mass lesion, when the bone flap is intentionally not replaced even when significant brain swelling is observed or anticipated [4]. There are two types of cases in which neurosurgeons choose to perform prophylactic DC: (1) prophylactic DC is planned before surgery because of severe brain edema seen on CT imaging and clinical evidence (e.g. if the degree of midline shift from early swelling was greater than the volume of a surgical amenable lesion) [16]; (2) prophylactic DC is decided at the time of evacuation of a mass lesion because of observed or anticipated severe brain edema (e.g. if surgeons encounter massive intraoperative brain swelling during evacuation of an acute subdural hematoma).

In most cases, DC is performed following the protocol for the treatment of refractory intracranial edema and hypertension as a secondary procedure. However, prophylactic DC should also play an important role in the management of severe TBI $[1,4]$. Moreover, it is important to bear in mind that ICP monitoring, and a number of therapies such as hypothermia, barbiturates coma, are not available for patients in all units at all times. DC which is readily available, inexpensive, and has only minor side effects, should play a much great role in the management of TBI in these circumstances [3].

The potential advantages of performing decompressive craniectomy as a primary form include: (1) it would rapidly reduce the elevated ICP and avoid the secondary ischemic and anoxic injury caused by existing or anticipative refractory intracranial hypertension; (2) it may reduce the mortality as a result of stopping refractory intracranial hypertension. However, it must be understood that the large craniectomy also puts the patient at the risk and cost of a second cranioplasty. In addition, primary decompressive craniectomy may also reduce the cost of intensive care during the acute phase of TBI, but then increase the whole cost of the secondary cranioplasty.

Table 3. Predictors of outcome

\begin{tabular}{lllllll}
\hline & Number & GCS $^{\Delta}$ & Age $^{\Delta}$ & $\begin{array}{l}\text { Sex } \\
\text { (male/female) }\end{array}$ & $\begin{array}{l}\text { Abnormal « } \\
\text { pupil response }\end{array}$ & $\begin{array}{l}\text { Midline shift « } \\
>5 \text { mm }\end{array}$ \\
\hline Group 1 & 44 & $4.5 \pm 0.9$ & $46.1 \pm 15.8$ & $31 / 13$ & $22 / 22(50 \%)$ & $38 / 6(86 \%)$ \\
Group 2 & 28 & $6.0 \pm 1.1$ & $46.4 \pm 15.6$ & $16 / 12$ & $5 / 23(18 \%)$ & $13 / 15(46 \%)$ \\
$P$ & & $<0.001$ & 0.932 & 0.312 & 0.007 & $<0.001$ \\
\hline
\end{tabular}

Group 1 = population of patients who had poor outcome, Group 2 = population of patients who had good outcome, removal of mass lesion (no/yes), abnormal pupil response (yes/no), midline shift $>5 \mathrm{~mm}$ (yes/no), $\Delta$ = independent Student $t$ test, $\boldsymbol{\Lambda}=$ Pearson chi-square 
Most studies of employment of DC in management of TBI included patients who were undergoing secondary or prophylactic DC. This makes it difficult to analyze these data in treatment of TBI. This study is distinct from previous studies in that it analyzes only primary DC. Controversy remains whether this surgical decompression should be performed as a secondary or prophylactic procedure. We believe that studying these two different DC approaches separately is a better way to understand their cost-benefit relationships. In this series involving 72 cases, cumulative deaths in the first 30 days after DC was 26\%, and 28 of 53 survivors had a good outcome. All of these patients had low GCS scores (no more than 8) at the time of admission of hospital. This suggests that most patients who underwent prophylactic DC had severe head trauma. This also suggests that patients undergoing prophylactic DC were a group likely to have a poorer prognosis than those usually undergoing secondary DC.

We found that factors including GCS score at admission, abnormal papillary responses, and midline shifts of greater than $5 \mathrm{~mm}$ were most important for the prediction of death in the first 30 days and the final outcome at 6 months after DC.

\section{Conclusion}

We reported a series of 72 patients who had severe head trauma and underwent prophylactic DC. Though there is still controversy of the final place of DC in the treatment of severe TBI, prophylactic performance of DC does play an important role in the management of highly elevated ICP, especially when other methods of reduction of ICP are unavailable. GCS score on admission, whether the patient had abnormal pupil response, and whether the midline shift was greater than $5 \mathrm{~mm}$ may be important prognostic factors related to final outcome. In addition, we suggest that in future studies of DC it is important to discuss prophylactic DC and secondary DC separately.

\section{Acknowledgement}

This study was supported by Grant 2007C33011 from the Zhejiang Science and Technology Bureau. The authors have no conflicts of interest to declare.

\section{References}

1. Maas AI, Dearden M, Teasdale GM, Braakman R, Cohadon F, Iannotti F, et al. EBIC-guidelines for management of severe head injury in adults. European Brain Injury Consortium. Acta Neurochir (Wien). 1997; 139:286-94.

2. The Brain Trauma Foundation. The American Association of Neurological Surgeons. The joint section on neurotrauma and critical care. Guidelines for cerebral perfusion pressure. J Neurotrauma. 2000; 17:507-11.

3. Piek J. Decompressive surgery in the treatment of traumatic brain injury. Curr Opin Crit Care. 2002; 8: 134-8.

4. Timofeev I, Hutchinson PJ. Outcome after surgical decompression of severe traumatic brain injury. Injury. 2006; 37:1125-32.

5. Cooper PR, Rovit RL, Ransohoff J. Hemicraniectomy in the treatment of acute subdural hematoma: a reappraisal. Surg Neurol. 1976; 5:25-8.

6. Gerl A, Tavan S. Bilateral craniectomy in the treatment of severe traumatic brain edema. Zentralbl Neurochir. 1980; 41:125-38.

7. Aarabi B, Hesdorffer DC, Ahn ES, Aresco C, Scalea TM, Eisenberg HM. Outcome following decompressive craniectomy for malignant swelling due to severe head injury. J Neurosurg. 2006; 104:469-79.

8. Guerra WK, Gaab MR, Dietz H, Mueller JU, Piek J, Fritsch MJ. Surgical decompression for traumatic brain swelling: indications and results. J Neurosurg. 1999; 90:187-96.

9. De Luca GP, Volpin L, Fornezza U, Cervellini P, Zanusso M, Casentini L, et al. The role of decompressive craniectomy in the treatment of uncontrollable posttraumatic intracranial hypertension. Acta Neurochir Suppl. 2000; 76:401-4.

10. Yang XF, Wen L, Shen F, Li G, Lou R, Liu WG, et al. Surgical complications secondary to decompressive craniectomy in patients with a head injury: a series of 108 consecutive cases. Acta Neurochir (Wien). 2008; 150:1241-7; discussion p. 1248.

11. Wardlaw JM, Easton VJ, Statham P. Which CT features help predict outcome after head injury? J Neurol Neurosurg Psychiatry. 2002; 72:188-92; discussion p. 151.

12. Treggiari MM, Schutz N, Yanez ND, Romand JA. Role of intracranial pressure values and patterns in predicting outcome in traumatic brain injury: a systematic review. Neurocrit Care. 2007; 6:104-12.

13. Huang SJ, Hong WC, Han YY, Chen YS, Wen CS, Tsan YS, et al. Clinical outcome of severe head injury in different protocol-driven therapies. J Clin Neurosci. 2007; 14:449-54. 
14. Stocchetti N, Zanaboni C, Colombo A, Citerio G, Beretta L, Ghisoni L, et al. Refractory intracranial hypertension and "second-tier" therapies in traumatic brain injury. Intensive Care Med. 2008; 34:461-7.

15. Chibbaro S, Tacconi L. Role of decompressive craniectomy in the management of severe head injury with refractory cerebral edema and intractable intracranial pressure. Our experience with 48 cases. Surg Neurol. 2007; 68:632-8.

16. Coplin WM, Cullen NK, Policherla PN, Vinas FC, Wilseck JM, Zafonte RD, et al. Safety and feasibility of craniectomy with duraplasty as the initial surgical intervention for severe traumatic brain injury. J Trauma. 2001; 50:1050-9. 